\title{
Risk Factors of Leishmania Infection among HIV-Infected Patients in Trang Province, Southern Thailand: A Study on Three Prevalent Species
}

\author{
Sakarn Charoensakulchai, ${ }^{1}$ Lertwut Bualert, ${ }^{2}$ Jipada Manomat, ${ }^{3}$ Mathirut Mungthin, ${ }^{1}$ Saovanee Leelayoova, ${ }^{1}$ Peerapan Tan- \\ ariya, ${ }^{3}$ Suradej Siripattanapipong, ${ }^{3}$ Tawee Naaglor, ${ }^{1}$ and Phunlerd Piyaraj ${ }^{1 *}$ \\ ${ }^{1}$ Department of Parasitology, Phramongkutklao College of Medicine, Bangkok, Thailand; ${ }^{2}$ Department of Medicine, Trang Hospital, Trang, \\ Thailand; ${ }^{3}$ Department of Microbiology, Faculty of Science, Mahidol University, Bangkok, Thailand
}

\begin{abstract}
There are two main species of Leishmania reported in Thailand, that is, Leishmania siamensis and Leishmania martiniquensis. Moreover, leishmaniasis cases caused by Leishmania donovani complex were also reported. There is still a lack of information concerning risk factors of Leishmania infection in Thailand. This study aimed to identify the risk factors of Leishmania infection caused by these three species among HIV-infected patients. A cross-sectional study was conducted in HIV clinic at Trang Hospital, Thailand. Nested PCR and sequencing were performed to detect Leishmania DNA in blood and saliva samples and identify Leishmania species. A standardized questionnaire was used to interview individuals. A total of 526 patients were recruited in this study. Sixty-three (12.0\%) were positive for L. siamensis, $24(4.6 \%)$ were positive for $L$. martiniquensis, and $23(4.4 \%)$ were positive for $L$. donovani complex. Risk factors of $L$. siamensis infection included using intravenous drug (adjusted odds ratio [AOR] 2.01, 95\% Cl: 1.01-4.02). Risk factors of L. martiniquensis infection included female gender (AOR 4.23, 95\% Cl: 1.52-11.75), using recreational drug (AOR 3.43, 95\% Cl: 1.00-11.74), and having comorbidities (AOR 4.94, 95\% Cl: 2.00-12.21). Risk factors of L. donovani complex infection included having opportunistic infection (AOR 4.22, 95\% Cl: 1.00-17.79), CD4 count 200-500 cells/mm ${ }^{3}$ (AOR $3.64,95 \% \mathrm{Cl}: 1.14-6.86$ ), and not using insect repellent (AOR 3.04, 95\% Cl: 1.08-8.58). This study identified the risk factors of Leishmania infection caused by three Leishmania species in Thailand. The data could be useful for disease prevention and control. Further studies on trends of Leishmania infection and preventive measures are recommended.
\end{abstract}

\section{INTRODUCTION}

Leishmania is a genus of the protozoan parasite, causes a disease called leishmaniasis, which ranges from chronic ulceration called cutaneous leishmaniasis (CL) to a fatal systemic disease called visceral leishmaniasis (VL). ${ }^{1}$ Leishmaniasis is transmitted by a bite of an infected sandfly that introduces metacyclic promastigotes, a motile form of the parasite, via the host's skin. ${ }^{1-3}$ The parasites are then phagocytosed by the host's macrophages ${ }^{4}$ where the promastigote shifts to the amastigote form, an immotile obligative intracellular stage, which multiplies and further invades other cells. ${ }^{4}$

There are more than 20 Leishmania species which are able to infect humans. ${ }^{5}$ In Thailand, most studies identified two novel species, Leishmania siamensis and Leishmania martiniquensis, as the main causes of leishmaniasis. ${ }^{6} \mathrm{~A}$ recent study reported Leishmania donovani complex, a group of Leishmania comprising L. donovani and Leishmania infantum, infections among HIV-infected patients as well. ${ }^{7}$ There was also a report of VL in non-HIV-infected patients caused by L. infantum in Thailand as well. ${ }^{8}$ Cutaneous leishmaniasis in L. martiniquensis infection could be ranging from single or multiple nodules to generalized non-ulcerative nodules, ulcerative lesions, or fibrotic lesions, of which the generalized form is called disseminated CL. ${ }^{6}$ On the other hand, the disseminated CL form of $L$. siamensis infection, which manifested as thick skin nodules, is rare. ${ }^{6}$ For VL, the presentations included prolonged fever, hepatosplenomegaly, weight loss, anemia, leukopenia, thrombocytopenia, and hypergammaglobulinemia, which are similar among $L$. martiniquensis, L. siamensis, and L. donovani complex. ${ }^{6,9}$

\footnotetext{
${ }^{*}$ Address correspondence to Phunlerd Piyaraj, Department of Parasitology, Phramongkutklao College of Medicine, 315 Rajawithee Rd., Thung Phyathai Sub-district, Rajadhewi District, Bangkok 10400, Thailand. E-mail: p_phunlerd@yahoo.com
}

Emergences of both VL and CL have been continuously reported in Thailand, especially in the southern provinces of the country. ${ }^{6,10-15}$ In 2010, the first case of VL infection in an HIV-infected patient in Thailand was reported. ${ }^{16}$ In 2015, Thailand was first considered as the endemic area of leishmaniasis. ${ }^{5,12}$

Risk factors of VL among HIV-infected patients in Thailand had already been addressed ${ }^{7}$; however, the differences of characteristics and risk factors of $L$. siamensis, $L$. martiniquensis, and $L$. donovani complex infections in HIV-infected patients are not yet identified. This study proposed to identify these differences among HIV-positive patients in Trang Province, an endemic area of leishmaniasis in southern Thailand. This information could be helpful in future diagnosis, determining disease progression, prevention of the disease, and control of vectors and reservoir hosts.

\section{MATERIAL AND METHODS}

Study design. This study is a continuity of the previously published study by Manomat et al. ${ }^{7}$ The current study was conducted in a cross-sectional design during February 2015 and February 2016 to identify the risk factors of Leishmania infection among HIV-infected patients who visited Trang Hospital, Trang Province, southern Thailand.

Study population and setting. Based on the study by Manomat et al., ${ }^{7}$ this study included HIV-infected patients who were aged 18 years or older and went to the HIV clinic in Trang Hospital. Trang Hospital is a major public tertiary care center of Trang Province and was classified by the Ministry of Public Health as a regional hospital. Trang Province is a coastal province in southern Thailand. It is located $837 \mathrm{~km}$ from Bangkok, the capital of Thailand. The climate was tropical monsoon with an average temperature of $27.2^{\circ} \mathrm{C}$ for the year. The average amount of precipitation for the year was 2,385.1 $\mathrm{mm}$. The highest precipitating month was September. 
The patients visited the HIV clinic every 6 months for followup test and received antiretroviral therapy. The patients lived in 10 districts of Trang Province, nine other provinces in southern Thailand, and three other provinces in other regions of Thailand. Information on the patients was retrieved from their medical records.

Ethical consideration. The research protocol was approved by the Ethical Committee of the Royal Thai Army Medical Department. The approval number was S017 h/56. Written consent was obtained before data collection. Personal information of the patients was disclosed and anonymized.

Samples collection and preparation. Blood samples of the patients were collected in an ethylenediaminetetraacetic acid (EDTA) anticoagulant tube. The amount of blood collected was $8 \mathrm{~mL}$. After collection, whole blood was centrifuged at $900 \times g$ for 10 minutes for separation of plasma and buffy coat. The centrifuged samples were preserved at $-20^{\circ} \mathrm{C}$. One-two milliliters of saliva sample of each patient was collected by spitting into sterile collection vials. Samples were preserved at $-20^{\circ} \mathrm{C}$ for $1-2$ hours until used for DNA detection.

Definitions. Leishmania infection was defined as an individual who was positive for Leishmania DNA from nested PCR using buffy coat and saliva samples, and, then, sequenced for species identification. Leishmania non-infection can be defined as an individual who was positive by neither direct antiglobulin test (DAT) nor nested PCR. Previous Leishmania infection can be defined as an individual who was infected with Leishmania and completely cured in the past, without relation with current Leishmania infection. These individuals were tested positive for DAT, but negative for nested PCR.

Detection of Leishmania antibodies. This study used DAT for screening patients with positive Leishmania infection. DATkit (KIT Biomedical Research, Amsterdam, the Netherlands) was used with the assay for Leishmania antibodies. The instruction was provided by the manufacturer. Positive control was retrieved from plasma of confirmed VL cases by the PCR method. Negative control was retrieved from plasma of healthy individuals. Positive titers were detected at value $\geq 1$ : 100. The cutoff value was recommended by the manufacturer. Patients' samples which were positive for Leishmania antibodies were further tested with PCR for species identification.

Detection of Leishmania DNA. Two hundred microliters of the buffy coat and saliva collected from each participant were extracted using Gen UPTM gDNA Kit (biotechrabbit, Hennigsdorf, Germany). The final volume of $40 \mu \mathrm{L}$ was eluted and preserved at $-20^{\circ} \mathrm{C}$. The nested PCR method was used for amplifying the internal transcribed spacer (ITS) 1 region on small subunit ribosomal DNA of the Leishmania. Primers LITSR and L5.8 were used for amplification of 319-348 amplicons during primary PCR process. ${ }^{17}$ For the secondary PCR process, two new primers were designed: LITSR2 and L5.8S inner; $25 \mu \mathrm{L}$ of PCR master mix for both buffy coat and saliva specimens comprised $12.5 \mathrm{pmol}$ of each primer, $0.2 \mathrm{mM}$ of dNTP (Promega, Madison, WI), $1.5 \mathrm{mM}$ of $\mathrm{MgCl}^{2}, 1 \times \mathrm{PCR}$ buffer, and $1 \mathrm{U}$ of Taq DNA polymerase (Promega, Madison, WI). Four and one microliters of DNA templates were used during primary and secondary reactions, respectively. Positive control was DNA of L. martiniquensis promastigotes. The overall processes consisted of pre-denaturation at $94^{\circ} \mathrm{C}$ for 3 minutes, 35 cycles of $94^{\circ} \mathrm{C}$ of denaturation for 1 minute, $54^{\circ} \mathrm{C}$ of annealing for 30 seconds, and $72^{\circ} \mathrm{C}$ of extension for 30 seconds. Final extension was performed at $72^{\circ} \mathrm{C}$ for 5 minutes. PCR products were separated by electrophoresis. Electrophoresis results were visualized by Molecular Imager Gel Doc XR + System with Imager Lab ${ }^{\mathrm{TM}} 3.0$ program (BioRad, Hercules, CA). The full detail of Leishmania DNA detection was described in study by Manomat et al. ${ }^{7}$

Sequence analysis. Positive PCR products were sent to U2Bio Co. Ltd. (Seoul, South Korea) for sequencing. BioEdit version 7.0.1 (Ibis Therapeutics, Carlsbad, CA) was used for validation of chromatogram. The sequences were multiplealigned with reference Leishmania strains from GenBank for species identification. The nested PCR results obtained from saliva samples were compared with those obtained from buffy coat samples. Phylogenetic tree was generated by neighborjoining method using MEGA program version 7.0 (Pennsylvania State University, University Park, PA).

Questionnaire. A standardized questionnaire was constructed. The questionnaire included demographic data, socioeconomic status, clinical symptoms, and risk behaviors. The data were collected by face-to-face interview.

Statistical analysis. Risk factors of L. siamensis, $L$. martiniquensis, and $L$. donovani complex infections were analyzed by univariate analysis. A statistical significance was indicated when $P<0.05$. Factors which were statistically significant in previous studies, statistically significant by univariate analysis, or $P<0.25$ by univariate analysis were recruited for multivariate analysis using "Enter" function. Odds ratio and $95 \% \mathrm{Cl}$ were calculated for both univariate and multivariate analyses. Factors which had $P<0.05$ by multivariate analysis were considered to be associate factors of $L$. siamensis, $L$. martiniquensis, and $L$. donovani complex. The program used for statistical analysis was STATA, version SE14 (Stata Corporation, College Station, TX).

\section{RESULTS}

A total of 724 patients with HIV infection were enrolled. From these, $643(88.8 \%)$ patients consented and answered the questionnaire. This study excluded 91 (12.6\%) people because of discordance between DAT (positive for Leishmania antibodies) and PCR (negative for species identification) results because these individuals were clarified as having previous infection. One case of Leishmania major infection and four cases of Leishmania lainsoni infection were identified, but excluded from the analysis because of limited number of cases and not being the common species found in Thailand. Twenty-one patients positive for Leishmania spp. were excluded because of species unidentification. As a result, 526 $(72.7 \%)$ patients were eligible for further analysis in this study.

Baseline characteristics of population. From 526 patients in this study, most were male (52.5\%) and worked as agricultural workers (31.2\%), and their age ranged from 40 to 50 years (46.0\%). The mean age was $43.85 \pm 0.37$ years. Clinical characteristics were as follows: $93.9 \%$ did not have opportunistic infections, $44.1 \%$ had lived with HIV for $5-10$ years, $78.1 \%$ had viral loads fewer than 50 copies $/ \mathrm{mL}, 70.7 \%$ had CD4 count greater than 500 cells $/ \mathrm{mL}$, and $75.5 \%$ did not have comorbidities. Risk factor characteristics included a majority of patients who had pets (50.2\%), did not live near forests (79.3\%), did not use either intravenous drug (82.1\%) or recreational drug (87.5\%), and did not use insect repellent $(77.8 \%)$, but used bed net (63.5\%). The data is displayed in Supplemental Table 1.

Identification of Leishmania species. A total of 110 (20.9\%) patients were infected with each of the three selected 
Leishmania species. Most patients were PCR positive from either buffy coat or saliva. However, some patients had overlapping positive PCR results from buffy coat and saliva. From nested PCR and sequencing results, patients infected with L. siamensis were 63 (12.0\%). Of these, 50 (9.5\%) were PCR positive from buffy coat, 24 (4.6\%) were PCR positive from saliva, and $11(2.1 \%)$ were PCR positive from both buffy coat and saliva. Twenty-four $(4.6 \%)$ patients were infected with $L$. martiniquensis, of which 17 (3.2\%) were PCR positive from buffy coat, $10(1.9 \%)$ were PCR positive from saliva, and three $(0.6 \%)$ were PCR positive from both buffy coat and saliva. There were $23(4.4 \%)$ patients who were infected with L. donovani complex of which $21(4.0 \%)$ were positive for buffy coat PCR and two $(0.4 \%)$ were positive for saliva PCR. No crossing between positive $L$. donovani complex infection from buffy coat and saliva PCRs were found.

Factors associated with the infection of each Leishmania species. Univariate and multivariate analyses for risk factors of $L$. siamensis infection are shown in Table 1. Leishmania siamensis infection was associated with using intravenous drug (adjusted odds ratio [AOR] 2.01, 95\% Cl: 1.01-4.02) after having adjusted for confounding factors with gender, age, having pets, CD4 counts, intravenous drug, recreational drug, using insect repellent, using bed net, and having comorbidities.

TABLE 1

Univariate and multivariate analyses for risk factors of $L$. siamensis infection among HIV patients in Trang Province, Thailand $(n=479)$

\begin{tabular}{|c|c|c|c|c|c|c|c|c|}
\hline \multirow[b]{2}{*}{ Characteristic } & \multicolumn{2}{|c|}{ Outcomes } & \multicolumn{3}{|c|}{ Univariate analysis } & \multicolumn{3}{|c|}{ Multivariate analysis } \\
\hline & $\begin{array}{l}\text { Uninfected with } L \text {. } \\
\text { siamensis, } n(\%)\end{array}$ & $\begin{array}{l}\text { Infected with } L \text {. } \\
\text { siamensis, } n(\%)\end{array}$ & $\begin{array}{l}\text { Crude } \\
\text { OR }\end{array}$ & $95 \% \mathrm{Cl}$ & $P$-value & $\begin{array}{l}\text { Adjusted } \\
\text { OR }\end{array}$ & $95 \% \mathrm{Cl}$ & $P$-value \\
\hline \multicolumn{9}{|l|}{ Gender } \\
\hline Male & 221 (86.3) & 35 (13.7) & 1.00 & - & - & 1.00 & - & - \\
\hline Female & $195(87.4)$ & $28(12.6)$ & 0.91 & $0.53-1.54$ & 0.719 & 0.88 & $0.50-1.56$ & 0.668 \\
\hline \multicolumn{9}{|l|}{ Age (years) } \\
\hline$<40$ & 142 (89.9) & $16(10.1)$ & 1.00 & - & - & 1.00 & - & - \\
\hline $40-50$ & $190(85.6)$ & $32(14.4)$ & 1.49 & $0.79-2.83$ & 0.217 & 1.35 & $0.69-2.65$ & 0.381 \\
\hline$>50$ & $84(84.8)$ & $15(15.2)$ & 1.58 & $0.75-3.37$ & 0.232 & 1.44 & $0.66-3.14$ & 0.365 \\
\hline \multicolumn{9}{|l|}{ Occupation } \\
\hline Unemployed & $40(87.0)$ & $6(13.0)$ & 1.00 & - & - & - & - & - \\
\hline Agricultural worker & 132 (89.8) & 15 (10.2) & 0.76 & $0.28-2.08$ & 0.590 & - & - & - \\
\hline Government officer & $30(81.1)$ & 7 (18.9) & 1.56 & $0.47-5.11$ & 0.466 & - & - & - \\
\hline Owning business & 82 (87.2) & $12(12.8)$ & 0.98 & $0.34-2.79$ & 0.963 & - & - & - \\
\hline Laborer & $91(85.1)$ & $16(14.9)$ & 1.17 & $0.43-3.22$ & 0.758 & - & - & - \\
\hline Others & $41(85.4)$ & $7(14.6)$ & 1.14 & $0.35-3.68$ & 0.829 & - & - & - \\
\hline \multicolumn{9}{|l|}{ Opportunistic infection } \\
\hline Yes & $23(82.1)$ & 5 (17.9) & 1.47 & $0.54-4.03$ & 0.450 & - & - & - \\
\hline No & $393(87.1)$ & 58 (12.9) & 1.00 & - & - & - & - & - \\
\hline \multicolumn{9}{|l|}{ Have pets } \\
\hline Yes & $210(89.0)$ & $26(11.0)$ & 0.69 & $0.40-1.18$ & 0.175 & 0.69 & $0.40-1.21$ & 0.198 \\
\hline No & $206(84.8)$ & $37(15.2)$ & 1.00 & - & - & 1.00 & - & - \\
\hline \multicolumn{9}{|c|}{ Forest located near residence } \\
\hline Yes & $84(87.5)$ & $12(12.5)$ & 0.93 & $0.47-1.82$ & 0.833 & - & - & - \\
\hline No & 332 (86.7) & $51(13.3)$ & 1.00 & - & - & - & - & - \\
\hline \multicolumn{9}{|l|}{ Years having HIV } \\
\hline$<5$ & $129(89.0)$ & $16(11.0)$ & 1.00 & - & - & - & - & - \\
\hline $5-10$ & 182 (85.9) & $30(14.2)$ & 1.33 & $0.70-2.54$ & 0.389 & - & - & - \\
\hline$>10$ & $105(86.1)$ & 17 (13.9) & 1.31 & $0.63-2.71$ & 0.474 & - & - & - \\
\hline \multicolumn{9}{|l|}{ Viral load (copies/mL) } \\
\hline$<50$ & $319(86.0)$ & $52(14.0)$ & 1.00 & - & - & - & - & - \\
\hline 50 and above & $97(89.8)$ & $11(10.2)$ & 0.70 & $0.35-1.39$ & 0.302 & - & - & - \\
\hline \multicolumn{9}{|l|}{ CD4 count (cells $/ \mathrm{mm}^{3}$ ) } \\
\hline$>500$ & 298 (86.9) & $45(13.1)$ & 1.00 & - & - & 1.00 & - & - \\
\hline $200-500$ & $80(87.0)$ & $12(13.0)$ & 0.99 & $0.50-1.97$ & 0.985 & 1.13 & $0.56-2.31$ & 0.734 \\
\hline$<200$ & $38(86.4)$ & $6(13.6)$ & 1.05 & $0.42-2.61$ & 0.924 & 1.24 & $0.48-3.20$ & 0.650 \\
\hline \multicolumn{9}{|l|}{ Intravenous drug use } \\
\hline Yes & 72 (82.7) & $15(17.2)$ & 1.49 & $0.79-2.81$ & 0.215 & 2.01 & $1.01-4.02$ & $0.047^{*}$ \\
\hline No & $344(87.8)$ & 48 (12.2) & 1.00 & - & - & 1.00 & - & - \\
\hline \multicolumn{9}{|l|}{ Recreational drug use } \\
\hline Yes & $54(90.0)$ & $6(10.0)$ & 0.71 & $0.29-1.72$ & 0.442 & 0.52 & $0.19-1.37$ & 0.183 \\
\hline No & 362 (86.4) & $57(13.6)$ & 1.00 & - & - & 1.00 & - & - \\
\hline \multicolumn{9}{|l|}{ Using insect repellent } \\
\hline Yes & $85(82.5)$ & $18(17.5)$ & 1.00 & - & - & 1.00 & - & - \\
\hline No & $331(88.0)$ & $45(12.0)$ & 0.64 & $0.35-1.17$ & 0.145 & 0.56 & $0.30-1.05$ & 0.070 \\
\hline \multicolumn{9}{|l|}{ Using bed net } \\
\hline Yes & 263 (86.2) & $42(13.8)$ & 1.00 & - & - & 1.00 & - & - \\
\hline No & 153 (87.9) & $21(12.1)$ & 0.86 & $0.67-2.04$ & 0.596 & 0.81 & $0.45-1.44$ & 0.468 \\
\hline \multicolumn{9}{|l|}{ Having comorbidities } \\
\hline Yes & $90(81.8)$ & $20(18.2)$ & 1.68 & $0.94-3.01$ & 0.078 & 1.66 & $0.89-3.08$ & 0.110 \\
\hline No & $326(88.4)$ & $43(11.7)$ & 1.00 & - & - & 1.00 & - & - \\
\hline
\end{tabular}

${ }^{*}$ Significant value at $95 \% \mathrm{Cl}$. 
Risk factors of $L$. martiniquensis infection included female gender (AOR 4.23, 95\% Cl: 1.52-11.75), using recreational drug (AOR 3.43, 95\% Cl: 1.00-11.74), and having comorbidities (AOR 4.94, 95\% Cl: 2.00-12.21). Adjusted confounding factors were gender, age, viral load, recreational drug use, and having comorbidities (Table 2 ).

Table 3 shows risk factors of $L$. donovani complex infection which included having opportunistic infection (AOR 4.22, 95\% Cl: 1.00-17.79), CD4 count 200-500 cells $/ \mathrm{mm}^{3}$ (AOR 3.64, 95\% Cl: 1.14-6.86), and not using insect repellent (AOR 3.04, 95\% Cl: 1.08-8.58) following adjustment for confounders with age, occupation, opportunistic infection, having pets, years having HIV, CD4 count, recreational drug use, and using insect repellent.

\section{DISCUSSION}

This study identified the risk factors of $L$. siamensis, $L$. martiniquensis, and $L$. donovani complex infections among HIV-infected patients who visited Trang Hospital, Trang, southern Thailand. This study included DNA detection from both blood and saliva samples. Although there was a study which had stated associated factors of Leishmania infection, ${ }^{7}$ there has never been a study which demonstrated associated factors of each individual species. Unlike other endemic areas

TABLE 2

Univariate and multivariate analyses for risk factors of $L$. martiniquensis infection among HIV patients in Trang Province, Thailand $(n=440)$

\begin{tabular}{|c|c|c|c|c|c|c|c|c|}
\hline \multirow[b]{3}{*}{ Characteristic } & \multicolumn{2}{|c|}{ Outcomes } & \multirow{2}{*}{\multicolumn{3}{|c|}{ Univariate analysis }} & \multirow{2}{*}{\multicolumn{3}{|c|}{ Multivariate analysis }} \\
\hline & \multirow{2}{*}{$\begin{array}{l}\text { Uninfected with L. } \\
\text { martiniquensis, } n(\%)\end{array}$} & \multirow{2}{*}{$\begin{array}{l}\text { Infected with } L \\
\text { martiniquensis, } n(\%)\end{array}$} & & & & & & \\
\hline & & & Crude OR & $95 \% \mathrm{Cl}$ & $P$-value & Adjusted OR & $95 \% \mathrm{Cl}$ & $P$-value \\
\hline \multicolumn{9}{|l|}{ Gender } \\
\hline Male & $221(96.9)$ & $7(3.1)$ & 1.00 & _- & _- & 1.00 & - & - \\
\hline Female & $195(92.0)$ & $17(8.0)$ & 2.75 & $1.12-6.78$ & $0.028^{\star}$ & 4.23 & $1.52-11.75$ & $0.006^{\star}$ \\
\hline \multicolumn{9}{|l|}{ Age (years) } \\
\hline$<40$ & $142(95.9)$ & $6(4.1)$ & 1.00 & - & - & 1.00 & - & - \\
\hline $40-50$ & $190(95.5)$ & $9(4.5)$ & 1.12 & $0.39-3.22$ & 0.832 & 0.75 & $0.24-2.31$ & 0.610 \\
\hline$>50$ & $84(90.3)$ & $9(9.7)$ & 2.54 & $0.87-7.38$ & 0.088 & 2.16 & $0.69-6.73$ & 0.184 \\
\hline \multicolumn{9}{|l|}{ Occupation } \\
\hline Unemployed & $40(97.7)$ & $1(2.4)$ & 1.00 & _- & - & - & - & - \\
\hline Agricultural worker & $132(89.8)$ & $10(7.0)$ & 3.03 & $0.38-24.40$ & 0.298 & - & - & - \\
\hline Government officer & $30(100.0)$ & $0(0.0)$ & - & - & - & _- & _- & _- \\
\hline Owning business & $82(94.3)$ & $5(5.8)$ & 2.44 & $0.28-21.58$ & 0.423 & - & - & - \\
\hline Laborer & $91(94.8)$ & $5(5.2)$ & 2.20 & $0.25-19.42$ & 0.479 & - & - & - \\
\hline Others & 41 (93.2) & $3(6.8)$ & 2.93 & $0.29-29.33$ & 0.361 & - & - & - \\
\hline \multicolumn{9}{|l|}{ Opportunistic infection } \\
\hline Yes & $23(95.8)$ & $1(4.2)$ & 0.74 & $0.96-5.75$ & 0.776 & - & - & - \\
\hline No & $393(94.5)$ & $23(5.5)$ & 1.00 & - & - & - & - & - \\
\hline \multicolumn{9}{|l|}{ Have pets } \\
\hline Yes & $210(94.2)$ & $13(5.8)$ & 1.16 & $0.51-2.65$ & 0.175 & - & - & - \\
\hline No & 206 (94.9) & $11(5.1)$ & 1.00 & - & - & _- & _- & _- \\
\hline \multicolumn{9}{|c|}{ Forest located near residence } \\
\hline Yes & $84(92.3)$ & $7(7.7)$ & 1.63 & $0.65-4.05$ & 0.295 & - & - & - \\
\hline No & $332(95.1)$ & $17(4.8)$ & 1.00 & - & - & - & - & - \\
\hline \multicolumn{9}{|l|}{ Years having HIV } \\
\hline$<5$ & $129(94.2)$ & $8(5.8)$ & 1.00 & _- & - & - & - & - \\
\hline $5-10$ & $182(94.3)$ & $11(5.7)$ & 0.97 & $0.38-2.49$ & 0.957 & - & - & - \\
\hline$>10$ & $105(95.5)$ & $5(4.6)$ & 0.77 & $0.24-2.42$ & 0.652 & - & - & - \\
\hline \multicolumn{9}{|l|}{ Viral load (copies/mL) } \\
\hline$<50$ & $318(93.5)$ & $22(6.5)$ & 1.00 & _- & _- & 1.00 & - & - \\
\hline 50 and above & $98(98.0)$ & $2(2.0)$ & 0.29 & $0.07-1.28$ & 0.102 & 0.25 & $0.05-1.14$ & 0.073 \\
\hline \multicolumn{9}{|l|}{ CD4 count (cells $/ \mathrm{mm}^{3}$ ) } \\
\hline$>500$ & $298(94.6)$ & $17(5.4)$ & 1.00 & - & - & - & - & - \\
\hline $200-500$ & $80(95.2)$ & $4(4.7)$ & 0.88 & $0.29-2.68$ & 0.985 & _- & _- & _- \\
\hline$<200$ & $38(92.7)$ & $3(7.3)$ & 1.38 & $0.39-4.94$ & 0.924 & - & - & - \\
\hline \multicolumn{9}{|l|}{ Intravenous drug use } \\
\hline Yes & $72(94.7)$ & $4(5.3)$ & 0.96 & $0.32-2.88$ & 0.936 & - & - & - \\
\hline No & $344(94.5)$ & $20(5.5)$ & 1.00 & $0.02-$ & - & _- & _ & _ \\
\hline \multicolumn{9}{|l|}{ Recreational drug use } \\
\hline Yes & $54(91.5)$ & $5(8.5)$ & 1.76 & $0.63-4.92$ & 0.278 & 3.43 & $1.00-11.74$ & $0.049^{\star}$ \\
\hline No & $362(95.0)$ & $19(5.0)$ & 1.00 & - & - & 1.00 & - & - \\
\hline \multicolumn{9}{|l|}{ Using insect repellent } \\
\hline Yes & $85(94.4)$ & $5(5.6)$ & 1.00 & - & _- & - & _- & - \\
\hline No & 331 (94.6) & $19(5.3)$ & 1.02 & $0.37-2.82$ & 0.962 & - & - & - \\
\hline \multicolumn{9}{|l|}{ Using bed net } \\
\hline Yes & $263(94.6)$ & $15(5.4)$ & 1.00 & _- & - & - & - & - \\
\hline No & $153(94.4)$ & $9(5.6)$ & 1.03 & $0.44-2.41$ & 0.943 & _ & _- & - \\
\hline Having comorbidities & & & & & & & & \\
\hline Yes & $90(87.4)$ & $13(13.6)$ & 4.28 & $1.86-9.87$ & $0.001^{*}$ & 4.94 & $2.00-12.21$ & $0.001^{*}$ \\
\hline No & $326(96.7)$ & $11(3.3)$ & 1.00 & - & - & 1.00 & _ & - \\
\hline
\end{tabular}

${ }^{*}$ Significant value at $95 \% \mathrm{Cl}$ 
TABLE 3

Univariate and multivariate analysis for risk factors of $L$. donovani complex infection among HIV patients in Trang Province, Thailand $(n=439)$

\begin{tabular}{|c|c|c|c|c|c|c|c|c|}
\hline \multirow[b]{2}{*}{ Characteristic } & \multicolumn{2}{|c|}{ Outcomes } & \multicolumn{3}{|c|}{ Univariate analysis } & \multicolumn{3}{|c|}{ Multivariate analysis } \\
\hline & $\begin{array}{l}\text { Uninfected with } L \text {. donovani } \\
\text { complex, } n(\%)\end{array}$ & $\begin{array}{l}\text { Infected with } L \text {. } \\
\text { donovani complex, } n(\%)\end{array}$ & $\begin{array}{c}\text { Crude } \\
\text { OR }\end{array}$ & $95 \% \mathrm{Cl}$ & $P$-value & $\begin{array}{l}\text { Adjusted } \\
\text { OR }\end{array}$ & $95 \% \mathrm{Cl}$ & $P$-value \\
\hline \multicolumn{9}{|l|}{ Gender } \\
\hline Male & $221(94.4)$ & $13(5.6)$ & 1.00 & - & - & - & - & - \\
\hline Female & $195(95.1)$ & $10(4.9)$ & 0.87 & $0.37-2.03$ & 0.751 & - & - & - \\
\hline \multicolumn{9}{|l|}{ Age (years) } \\
\hline$<40$ & $142(93.4)$ & $10(6.6)$ & 1.00 & - & - & 1.00 & - & - \\
\hline $40-50$ & $190(94.5)$ & $11(5.5)$ & 0.82 & $0.34-1.99$ & 0.664 & 0.55 & $0.19-1.54$ & 0.545 \\
\hline$>50$ & 84 (97.7) & 2 (2.3) & 0.34 & $0.07-1.58$ & 0.168 & 0.35 & $0.07-1.75$ & 0.348 \\
\hline \multicolumn{9}{|l|}{ Occupation } \\
\hline Unemployed & 40 (97.6) & $1(2.4)$ & 1.00 & - & - & 1.00 & - & - \\
\hline Agricultural worker & $132(95.0)$ & $7(5.0)$ & 2.12 & $0.25-17.76$ & 0.488 & 1.38 & $0.14-13.48$ & 0.783 \\
\hline $\begin{array}{l}\text { Government } \\
\text { officer }\end{array}$ & $30(100.0)$ & $0(0.0)$ & - & - & - & - & - & - \\
\hline Owning business & 82 (93.2) & $6(6.8)$ & 2.93 & $0.34-25.14$ & 0.328 & 2.79 & $0.29-27.19$ & 0.376 \\
\hline Laborer & 91 (91.9) & $8(8.1)$ & 3.52 & $0.43-29.06$ & 0.243 & 3.34 & $0.36-31.56$ & 0.291 \\
\hline Others & $41(97.6)$ & $1(2.4)$ & 0.98 & $0.06-16.14$ & 0.986 & 0.89 & $0.05-16.81$ & 0.935 \\
\hline \multicolumn{9}{|l|}{ Opportunistic infection } \\
\hline Yes & $23(88.5)$ & $3(11.5)$ & 2.56 & $0.71-9.26$ & 0.151 & 4.22 & $1.00-17.79$ & $0.050 *$ \\
\hline No & 393 (95.2) & $20(4.8)$ & 1.00 & - & - & 1.00 & - & - \\
\hline \multicolumn{9}{|l|}{ Have pets } \\
\hline Yes & $210(94.2)$ & $15(6.7)$ & 1.84 & $0.76-4.43$ & 0.175 & 1.54 & $0.59-4.06$ & 0.381 \\
\hline No & 206 (96.3) & $8(3.7)$ & 1.00 & - & - & 1.00 & - & - \\
\hline \multicolumn{9}{|c|}{ Forest located near residence } \\
\hline Yes & $84(93.3)$ & $6(6.8)$ & 1.39 & $0.53-3.65$ & 0.497 & - & - & - \\
\hline No & $332(95.1)$ & $17(4.8)$ & 1.00 & - & - & - & - & - \\
\hline \multicolumn{9}{|l|}{ Years having HIV } \\
\hline$<5$ & $129(96.3)$ & $5(3.7)$ & 1.00 & - & - & 1.00 & - & - \\
\hline $5-10$ & $182(95.3)$ & $9(4.7)$ & 1.28 & $0.42-3.90$ & 0.669 & 1.79 & $0.53-6.09$ & 0.349 \\
\hline$>10$ & $105(92.1)$ & $9(7.9)$ & 2.21 & $0.79-6.80$ & 0.166 & 2.58 & $0.75-8.86$ & 0.132 \\
\hline \multicolumn{9}{|l|}{ Viral load (copies/mL) } \\
\hline$<50$ & $319(94.7)$ & $18(5.3)$ & 1.00 & - & - & - & - & - \\
\hline \multirow{2}{*}{\multicolumn{9}{|c|}{ CD4 count (cells $/ \mathrm{mm}^{3}$ ) }} \\
\hline & & & & & & & & \\
\hline$>500$ & $298(96.1)$ & $12(3.8)$ & 1.00 & - & - & 1.00 & - & - \\
\hline $200-500$ & 80 (89.9) & $9(10.1)$ & 2.79 & $1.14-6.86$ & $0.025^{*}$ & 3.64 & $1.29-10.28$ & $0.015^{\star}$ \\
\hline$<200$ & $38(95.0)$ & $2(5.0)$ & 1.38 & $0.28-6.06$ & 0.732 & 1.97 & $0.37-10.50$ & 0.425 \\
\hline \multicolumn{9}{|l|}{ Intravenous drug use } \\
\hline Yes & $72(96.0)$ & $3(4.0)$ & 0.72 & $0.21-2.48$ & 0.598 & - & - & - \\
\hline No & $344(94.5)$ & $20(5.5)$ & 1.00 & - & - & - & - & - \\
\hline \multicolumn{9}{|l|}{ Recreational drug use } \\
\hline Yes & 54 (98.2) & $1(1.8)$ & 0.30 & $0.04-2.31$ & 0.250 & 0.24 & $0.03-1.97$ & 0.184 \\
\hline No & 362 (94.3) & $22(5.7)$ & 1.00 & - & - & 1.00 & - & - \\
\hline \multicolumn{9}{|l|}{ Using insect repellent } \\
\hline Yes & 85 (90.4) & $9(9.6)$ & 1.00 & - & - & 1.00 & - & - \\
\hline No & 331 (95.9) & $14(4.1)$ & 0.40 & $0.17-0.95$ & $0.039^{*}$ & 3.04 & $1.08-8.58$ & $0.036^{\star}$ \\
\hline \multicolumn{9}{|l|}{ Using bed net } \\
\hline Yes & 263 (94.9) & $14(5.1)$ & 1.00 & - & - & - & - & \\
\hline No & $153(94.4)$ & 9 (5.6) & 1.11 & $0.47-2.61$ & 0.820 & - & - & - \\
\hline \multicolumn{9}{|l|}{ Having comorbidities } \\
\hline Yes & $90(93.8)$ & $6(6.3)$ & 1.28 & $0.49-3.34$ & 0.616 & - & - & - \\
\hline No & $326(95.0)$ & $17(5.0)$ & 1.00 & - & - & - & - & - \\
\hline
\end{tabular}

is $=$ Leishmania donovani $\mathrm{OR}=$ odds ratio.

* Significant value at $95 \% \mathrm{Cl}$.

where only single Leishmania species was dominantly found, ${ }^{18-21}$ Thailand, as an endemic area, harbored multiple species detection. ${ }^{6,22}$ Therefore, it is crucial to address varying associated factors contributed to infection of different Leishmania species.

This study reported an association between $L$. siamensis and intravenous drug use. There were sparse and unproven theories of such association, but not unprecedented. ${ }^{23-26}$ Previous studies also showed that Leishmania parasites could be transmitted via blood transfusion from healthy seropositive donors to recipients. ${ }^{27-30}$ A study from Madrid, Spain, found that the parasites can be found in clotted blood lodged in the syringes shared by various users. ${ }^{23}$ As Leishmania's life cycle included the amastigote form, which inoculates in hosts' macrophage circulating in blood vessels by sharing intravenous syringes, the artificial life cycle of Leishmania is formed. ${ }^{31}$ Through this mode, the remaining amastigoteinfected macrophages in the shared syringes were transmitted from infected individuals to others. ${ }^{32}$ Support of this theory was statistically reported as intravenous drug use accounted to be one of the risks of HIV-associated leishmaniasis in Spain. ${ }^{26}$ Because the study in Seville, Spain, reported that the species infected among intravenous drug users was $L$. infantum, ${ }^{33}$ this study hypothesized that there 
were some degrees of genetic and biologic resemblance between $L$. infantum and $L$. siamensis which affected their greater transmission from infected individuals to others via the route of injected syringes more than other species.

Leishmania martiniquensis infection was more prevalent and associated with female gender in this study, which contrasted to other studies. ${ }^{34-36}$ However, some studies reported females being equally to or having higher prevalent of males with leishmaniasis. ${ }^{37,38}$ A study in Kabul, Afghanistan, identified female gender as an associated factor of Leishmania tropica infection. ${ }^{39}$ Although an earlier experimental study showed that there was no significant difference between female and male genders toward sandfly attractiveness, ${ }^{40}$ the exposure of females and males to sandfly was still inconclusive by epidemiological study because there were limited sources. A previous study showed that the associated factors of Leishmania infection in Thailand might include living in stilt houses, an architectural feature common in southern Thailand where floods and monsoons occurred frequently. ${ }^{7}$ Some females were more likely to be bound to house works and, thus, have more chances of exposure to sandflies. ${ }^{41}$

There were no reports of a direct association between recreational drug use and Leishmania infection. This study assumed that there was an indirect relationship between recreational drug use and Leishmania infection via increasing risk of HIV infection. Recreational drug use is highly associated with risks of HIV infection in various modes such as interaction between recreational drugs and anti-HIV agents ${ }^{42}$ and increasing risky sexual behaviors. ${ }^{43,44}$ As a result, leishmaniasis, as an opportunistic infection associated with HIV infection because of impaired immunity, ${ }^{45-48}$ was indirectly associated with recreational drug use. Moreover, this study indicated the association between existence of comorbid illnesses and $L$. martiniquensis in HIV-infected patients. Antiretroviral therapy used for HIV treatment was usually linked to increased risks of comorbidities such as diabetes mellitus and dyslipidemia. ${ }^{49-51}$ In various ways, such comorbidities interfere with immune responses and resulted in susceptibilities of opportunistic infections, including Leishmania infection. ${ }^{52-54}$

In this study, L. donovani complex included $L$. donovani and $L$. infantum. These two species were grouped together as $L$. donovani complex because of their proximities that the differentiation of the ITS1 region was difficult. ${ }^{55}$ Leishmania donovani complex infections could be considered an emerging infection in Thailand because there had been very limited report of the infection. ${ }^{56}$ However, there was a report that cats were reservoir host of $L$. donovani complex in southern Thailand. ${ }^{57}$ As a result, domestic infection was not impossible.

Associated factors of $L$. donovani complex infections included having opportunistic infections and CD4 between 200 and 500 cells $/ \mathrm{mm}^{3}$ in this study. Patients with lower CD4 might have acquired opportunistic infections easier than those with high CD4. ${ }^{58}$ Association between CD4 200-500 cells $/ \mathrm{mm}^{3}$ and $L$. donovani complex infection could be explained with data from a previous study, stating that $L$. donovani complex was associated with VL relapse in HIV-infected patients with CD4 greater than 200 cells $/ \mathrm{mm}^{3}$, which is clinically latent, because of virulence of the species. ${ }^{59}$ Visceral leishmaniasis was also found to deteriorate immunological recovery of HIVinfected patients which increased risks of acquiring opportunistic infections. ${ }^{60}$ Thus, this study suspected correlation between $L$. donovani complex infections which accompanied other opportunistic infections even in latent HIV-infected patients because of species' virulence. This study did not find a relationship between $L$. donovani complex infections and CD4 level lower than 200 cells $/ \mathrm{mm}^{3}$ because of the limited number of cases positive with $L$. donovani complex infections. The small number of cases could be subjected to the high efficacy of HIV treatment which, in turn, reduced the incidence of leishmaniasis in patients with a low CD4 level. ${ }^{61}$

It was found that patients with no insect repellent usage were more likely to be infected with $L$. donovani complex significantly. Previous studies reported a lower incidence of leishmaniasis in individuals who used insect repellent, insecticidal control, or permethrin-impregnated cloth because of reduced risks of sandfly bite in both human and animal studies. ${ }^{62-65}$

This study contained some limitations. First, there were only three species which were studied. Other isolated species including $L$. major and L. lainsoni were omitted from this study because of their limited number of cases. Unidentified species were also excluded from the analysis. It is suggested that further studies on the prevalence of these species in other regions of Thailand should be processed. Second, although this study separately analyzed associated factors of infection by each of three species, some factors could not solely be explained by epidemiological studies. As stated in the statement, this study suspected that there were some degrees of genetic and biologic factors of both the parasite and host, which influenced infection of specific species more than others. Some of these factors, such as survival of the parasites in discarded syringes and clotted blood and virulence of each species in immunocompromised hosts, needed thorough investigations in further studies to establish theories of their infections relating to genetic and biologic factors.

This study identified the risk factors of infection of three Leishmania species prevalent in Thailand among HIV-infected patients. Several factors identified in this study should be dealt with thoroughly, such as elimination of illegal intravenous and recreational drugs use, effective control and monitoring of comorbid illnesses in HIV-infected individuals, and prevention of opportunistic infections in HIV-infected patients and vector controls by either insecticidal or repellent methods in endemic areas. It is recommended that further studies on leishmaniasis in other regions of Thailand due to different environmental factors should be processed.

Received April 22, 2020. Accepted for publication June 15, 2020.

Published online July 20, 2020.

Note: Supplemental table appears at www.ajtmh.org.

Acknowledgments: We express our appreciation to all staff and participants at Trang Hospital for their support of this study.

Financial support: This study was funded by the Phramongkutklao College of Medicine fund.

Authors' addresses: Sakarn Charoensakulchai, Mathirut Mungthin, Saovanee Leelayoova, Tawee Naaglor, and Phunlerd Piyaraj, Department of Parasitology, Phramongkutklao College of Medicine, Bangkok, Thailand, E-mails: sakarn.ch@pcm.ac.th, mathirut@ hotmail.com, s_leelayoova@scientist.com, taweenaaglor@yahoo.com, and p_phunlerd@yahoo.com. Lertwut Bualert, Department of Medicine, Trang Hospital, Trang, Thailand, E-mail: dr_lertwut_clinic@yahoo.com. Jipada Manomat, Peerapan Tan-ariya, and Suradej Siripattanapipong, Department of Microbiology, Faculty of Science, Mahidol University, Bangkok, Thailand, E-mails: manomat.j@gmail.com, peerapan.tan@ mahidol.ac.th, and suradejs@rocketmail.com. 


\section{REFERENCES}

1. Handman E, 1999. Cell biology of Leishmania. Adv Parasitol 44: 1-39.

2. Killick-Kendrick $R, 1990$. The life-cycle of Leishmania in the sandfly with special reference to the form infective to the vertebrate host. Ann Parasitol Hum Comp 65: 37-42.

3. Gossage SM, Rogers ME, Bates PA, 2003. Two separate growth phases during the development of Leishmania in sand flies: implications for understanding the life cycle. Int $J$ Parasitol 33: 1027-1034.

4. Handman E, Bullen DV, 2002. Interaction of Leishmania with the host macrophage. Trends Parasitol 18: 332-334.

5. World Health Organization, 2017. Global leishmaniasis update, 2006-2015: a turning point in leishmaniasis surveillance-le point sur la situation mondiale de la leishmaniose, 2006-2015: un tournant dans la surveillance de la maladie. Wkly Epidemiol Rec 92: 557-565.

6. Leelayoova S, Siripattanapipong S, Manomat J, Piyaraj P, TanAriya P, Bualert L, Mungthin M, 2017. Leishmaniasis in Thailand: a review of causative agents and situations. Am J Trop Med Hyg 96: 534-542.

7. Manomat J, Leelayoova S, Bualert L, Tan-ariya P, Siripattanapipong S, Mungthin M, Naaglor T, Piyaraj P, 2017. Prevalence and risk factors associated with Leishmania infection in Trang Province, southern Thailand. PLoS Negl Trop Dis 11: e0006095.

8. Maharom $P$, Siripattanapipong $S$, Mungthin $M$, Naaglor $T$, Sukkawee R, Wattana W, Wanachiwanawin D, Areechokchai D, Leelayoova S, 2008. Visceral leishmaniasis caused by Leishmania infantum in Thailand. Southeast Asian J Trop Med Public Health 39: 988-990.

9. Sundar S, Rai M, 2002. Laboratory diagnosis of visceral leishmaniasis. Clin Diagn Lab Immunol 9: 951-958.

10. Hotez PJ, Bottazzi ME, Strych U, Chang L-Y, Lim YA, Goodenow MM, AbuBakar S, 2015. Neglected tropical diseases among the Association of Southeast Asian Nations (ASEAN): overview and update. PLoS Negl Trop Dis 9: e0003575.

11. Kongkaew W, Siriarayaporn P, Leelayoova S, Supparatpinyo K, Areechokchai D, Duang-ngern P, Chanachai K, Sukmee T, Samung Y, Sridurongkathum P, 2007. Autochthonous visceral leishmaniasis: a report of a second case in Thailand. Southeast Asian J Trop Med Public Health 38: 8.

12. Sukmee $T$, Siripattanapipong $S$, Mungthin $M$, Worapong J, Rangsin R, Samung $\mathrm{Y}$, Kongkaew W, Bumrungsana K, Chanachai K, Apiwathanasorn C, 2008. A suspected new species of Leishmania, the causative agent of visceral leishmaniasis in a Thai patient. Int J Parasito/ 38: 617-622.

13. Kattipathanapong $P$, Akaraphanth $R$, Krudsood S, Riganti $M$, Viriyavejakul P, 2012. The first reported case of autochthonous cutaneous leishmaniasis in Thailand. Southeast Asian $J$ Trop Med Public Health 43: 17-20.

14. Cotton JA, 2017. The expanding world of human leishmaniasis. Trends Parasitol 33: 341-344.

15. Bualert L, Charungkiattikul W, Thongsuksai $P$, Mungthin $M$, Siripattanapipong S, Khositnithikul R, Naaglor T, Ravel C, El Baidouri F, Leelayoova S, 2012. Autochthonous disseminated dermal and visceral leishmaniasis in an AIDS patient, southern Thailand, caused by Leishmania siamensis. Am J Trop Med Hyg 86: 821-824.

16. Suankratay C, Suwanpimolkul G, Wilde H, Siriyasatien P, 2010. Autochthonous visceral leishmaniasis in a human immunodeficiency virus (HIV)-infected patient: the first in Thailand and review of the literature. Am J Trop Med Hyg 82: 4-8.

17. El Tai NO, El Fari M, Mauricio I, Miles MA, Oskam L, El Safi SH, Presber WH, Schönian G, 2001. Leishmania donovani: intraspecific polymorphisms of Sudanese isolates revealed by PCR-based analyses and DNA sequencing. Exp Parasitol 97: 35-44.

18. Miranda A, Carrasco R, Paz H, Pascale JM, Samudio F, Saldaña A, Santamaría G, Mendoza Y, Calzada JE, 2009. Molecular epidemiology of American tegumentary leishmaniasis in Panama. Am J Trop Med Hyg 81: 565-571.

19. Rougeron V, De Meeûs T, Hide M, Waleckx E, Bermudez $H$, Arevalo J, Llanos-Cuentas A, Dujardin J-C, De Doncker S, Le
Ray D, 2009. Extreme inbreeding in Leishmania braziliensis. Proc Natl Acad Sci USA 106: 10224-10229.

20. Ready PD, 2014. Epidemiology of visceral leishmaniasis. Clin Epidemiol 6: 147.

21. Sharifi F, Sharifi I, Zarean M, Parizi MH, Aflatoonian M, Harandi MF, Zahmatkesh R, Mashayekhi M, Kermanizadeh A, 2012. Spatial distribution and molecular identification of Leishmania species from endemic foci of south-eastern Iran. Iran J Parasitol 7: 45.

22. Siriyasatien $P$, Chusri S, Kraivichian K, Jariyapan N, Hortiwakul T, Silpapojakul K, Pym AM, Phumee A, 2016. Early detection of novel Leishmania species DNA in the saliva of two HIV-infected patients. BMC Infect Dis 16: 89.

23. Cruz I, Morales M, Noguer I, Rodriguez A, Alvar J, 2002. Leishmania in discarded syringes from intravenous drug users. Lancet 359: 1124-1125.

24. Pineda J, Macías J, Morillas F, Fernández-Ochoa J, Cara J, de La Rosa R, Mira J, Martín-Sánchez J, González M, Delgado J, 2001. Evidence of increased risk for Leishmania infantum infection among HIV-seronegative intravenous drug users from southern Spain. Eur J Clin Microbiol Infect Dis 20: 354-357.

25. Alexandrino-de-Oliveira $P$, Santos-Oliveira JR, Dorval MEC, DaCosta FdCB, Pereira GROL, Cunha RVd, Paniago AMM, DaCruz AM, 2010. HIV/AIDS-associated visceral leishmaniasis in patients from an endemic area in Central-west Brazil. Mem Inst Oswaldo Cruz 105: 692-697.

26. Colebunders R, Depraetere K, Verstraeten T, Lambert J, Hauben E, Van Marck E, Maurer T, Bañuls A-L, Dujardin J-C, 1999. Unusual cutaneous lesions in two patients with visceral leishmaniasis and HIV infection. J Am Acad Dermatol 41: 847-850.

27. Otero A, da Silva VO, Luz KG, Palatnik M, Pirmez C, Fernandes O, de Sousa CP, 2000. Occurrence of Leishmania donovani DNA in donated blood from seroreactive Brazilian blood donors. Am $J$ Trop Med Hyg 62: 128-131.

28. Dey A, Singh S, 2006. Transfusion transmitted leishmaniasis: a case report and review of literature. Indian J Med Microbiol 24: 165.

29. Luz KG, Da Silva VO, Gomes EM, Machado FC, Araujo MA, Fonseca HE, Freire TC, d'Almeida JB, Palatnik M, Palatnik-deSousa CB, 1997. Prevalence of anti-Leishmania donovani antibody among Brazilian blood donors and multiply transfused hemodialysis patients. Am J Trop Med Hyg 57: 168-171.

30. le Fichoux Y, Quaranta J-F, Aufeuvre J-P, Lelievre A, Marty P, Suffia I, Rousseau D, Kubar J, 1999. Occurrence of Leishmania infantum parasitemia in asymptomatic blood donors living in an area of endemicity in southern France. $J$ Clin Microbiol 37: 1953-1957.

31. Burton A, 2001. Sharing needles may produce artificial leishmaniasis cycle. Lancet Infect Dis 1: 4.

32. Molina R, Gradoni L, Alvar J, 2003. HIV and the transmission of Leishmania. Ann Trop Med Parasitol 97: 29-45.

33. Pineda JA, Martin-Sanchez J, Macías J, Morillas F, 2002. Leishmania spp infection in injecting drug users. Lancet 360 : 950-951.

34. Jeronimo SM, Duggal P, Braz RF, Cheng C, Monteiro GR, Nascimento ET, Martins DR, Karplus TM, Ximenes MF, Oliveira CC, 2004. An emerging peri-urban pattern of infection with Leishmania chagasi, the protozoan causing visceral leishmaniasis in northeast Brazil. Scand J Infect Dis 36: 443-449.

35. Belo VS, Werneck GL, Barbosa DS, Simões TC, Nascimento BWL, da Silva ES, Struchiner CJ, 2013. Factors associated with visceral leishmaniasis in the Americas: a systematic review and meta-analysis. PLoS Negl Trop Dis 7: e2182.

36. Machado-Coelho GL, Caiaffa WT, Genaro O, Magalhaes PA, Mayrink W, 2005. Risk factors for mucosal manifestation of American cutaneous leishmaniasis. Trans $R$ Soc Trop Med Hyg 99: 55-61.

37. Ahluwalia IB, Bern C, Wagatsuma Y, Costa C, Chowdhury R, Ali M, Amann J, Haque R, Breiman R, Maguire JH, 2004. Visceral leishmaniasis: consequences to women in a Bangladeshi community. J Womens Health (Larchmt) 13: 360-364.

38. Velez I, Hendrickx E, Robledo S, 2001. Gender and cutaneous leishmaniasis in Colombia. Cad Saude Publica 17: 171-180. 
39. Reithinger R, Mohsen M, Aadil K, Sidiqi M, Erasmus P, Coleman PG, 2003. Anthroponotic cutaneous leishmaniasis, Kabul, Afghanistan. Emerg Infect Dis 9: 727.

40. Rebollar-Tellez E, Hamilton J, Ward R, 1999. Response of female Lutzomyia longipalpis to host odour kairomones from human skin. Physiol Entomol 24: 220-226.

41. Al-Kamel MA, 2016. Impact of leishmaniasis in women: a practical review with an update on my ISD-supported initiative to combat leishmaniasis in Yemen (ELYP). Int J Womens Dermatol 2: 93-101.

42. Antoniou T, Tseng AL-i, 2002. Interactions between recreational drugs and antiretroviral agents. Ann Pharmacother 36: 1598-1613.

43. Xu J-J, Qian H-Z, Chu Z-X, Zhang J, Hu Q-H, Jiang Y-J, Geng $W-Q$, Zhang $C M$, Shang $H, 2014$. Recreational drug use among Chinese men who have sex with men: a risky combination with unprotected sex for acquiring HIV infection. Biomed Res Int 2014: 725361.

44. Halkitis PN, Parsons JT, 2002. Recreational drug use and HIV-risk sexual behavior among men frequenting gay social venues. $J$ Gay Lesbian Soc Serv 14: 19-38.

45. Pasquau F, Ena J, Sanchez R, Cuadrado J, Amador C, Flores J, Benito C, Redondo C, Lacruz J, Abril V, 2005. Leishmaniasis as an opportunistic infection in HIV-infected patients: determinants of relapse and mortality in a collaborative study of 228 episodes in a Mediterranean region. Eur J Clin Microbiol Infect Dis 24: 411-418.

46. Pintado V, Lopez-Velez R, 2001. HIV-associated visceral leishmaniasis. Clin Microbiol Infect 7: 291-300.

47. Pintado V, Martin-Rabadan P, Rivera ML, Moreno S, Bouza E, 2001. Visceral leishmaniasis in human immunodeficiency virus (HIV)-infected and non-HIV-infected patients: a comparative study. Medicine 80: 54-73.

48. Laguna F, Adrados M, Alvar J, Soriano V, Valencia M, Moreno V, Polo R, Verdejo J, Jimenez M, Martinez P, 1997. Visceral leishmaniasis in patients infected with the human immunodeficiency virus. Eur J Clin Microbiol Infect Dis 16: 898-903.

49. Kalra S, Agrawal N, 2013. Diabetes and HIV: current understanding and future perspectives. Curr Diab Rep 13: 419-427.

50. Kalra S, Kalra B, Agrawal N, Unnikrishnan A, 2011. Understanding diabetes in patients with HIV/AIDS. Diabetol Metab Syndr 3: 2.

51. Neto P, da Silva LF, Neves MBd, Ribeiro-Rodrigues R, Page K, Miranda AE, 2013. Dyslipidemia and fasting glucose impairment among HIV patients three years after the first antiretroviral regimen in a Brazilian AIDS outpatient clinic. Braz $\mathrm{J}$ Infect Dis 17: 438-443.

52. Faria MS, Reis FC, Lima APC, 2012. Toll-like receptors in Leishmania infections: guardians or promoters? J Parasitol Res 2012: 930257.
53. Shamshiev AT, Ampenberger F, Ernst B, Rohrer L, Marsland BJ, Kopf M, 2007. Dyslipidemia inhibits toll-like receptor-induced activation of CD8 $\alpha$-negative dendritic cells and protective Th1 type immunity. J Exp Med 204: 441-452.

54. Schwetz V, Trummer C, Friedl C, Beham-Schmid C, Kulnik R, Wölfler A, Horvath K, Wunsch S, Prattes J, Zollner-Schwetz I, 2018. Visceral leishmaniasis in a patient with diabetes mellitus type 2 and discrete bicytopenia. Clin Case Rep 6: 78.

55. Schönian G, Nasereddin A, Dinse N, Schweynoch C, Schallig HD, Presber W, Jaffe CL, 2003. PCR diagnosis and characterization of Leishmania in local and imported clinical samples. Diagn Microbiol Infect Dis 47: 349-358.

56. Pandey $\mathrm{N}$ et al., 2018. Detection of Leishmania DNA in saliva among patients with HIV/AIDS in Trang Province, southern Thailand. Acta Trop 185: 294-300.

57. Nimsuphan B, Chimnoi W, Kengradomkij C, Pangjai D, Jiyipong T, Khomkao J, Cheevasareechon M, Jirasutas W, 2014. Detection of anti-Leishmania donovani complex antibodies of dogs and cats from Southern Thailand. KKU Vet J 24: 9-19.

58. Ghate M, Deshpande S, Tripathy S, Nene M, Gedam P, Godbole S, Thakar M, Risbud A, Bollinger R, Mehendale S, 2009. Incidence of common opportunistic infections in HIV-infected individuals in Pune, India: analysis by stages of immunosuppression represented by CD4 counts. Int J Infect Dis 13: e1-e8.

59. Cota GF, De Sousa MR, Rabello A, 2011. Predictors of visceral leishmaniasis relapse in HIV-infected patients: a systematic review. PLoS Negl Trop Dis 5.

60. Lopez-Velez R, 2003. The impact of highly active antiretroviral therapy (HAART) on visceral leishmaniasis in Spanish patients who are co-infected with HIV. Ann Trop Med Parasit 97: 143-147.

61. López-Vélez R, Casado J, Pintado V, 2001. Decline of a visceral leishmaniasis epidemic in HIV-infected patients after the introduction of highly active antiretroviral therapy (HAART). Clin Microbiol Rev 7: 394-395.

62. Soto J, Medina F, Dember N, Berman J, 1995. Efficacy of permethrin-impregnated uniforms in the prevention of malaria and leishmaniasis in Colombian soldiers. Clin Infect Dis 21: 599-602.

63. Maroli M, Khoury C, 2004. Prevention and control of leishmaniasis vectors: current approaches. Parassitologia 46: 211-215.

64. Maroli M, Gradoni L, Oliva G, Castagnaro M, Crotti A, Lubas G, Paltrinieri S, Roura X, Zini E, Zatelli A, 2010. Guidelines for prevention of leishmaniasis in dogs. J Am Vet Med Assoc 236: $1200-1206$.

65. Gonzalez U, Pinart M, Sinclair D, Firooz A, Enk C, Vélez ID, Esterhuizen TM, Tristan M, Alvar J, 2015. Vector and reservoir control for preventing leishmaniasis. Cochrane Database Syst Rev 2015: CD008736. 